\title{
Estimation of the Reception Angle Distribution Based on the Power Delay Spectrum or Profile
}

\author{
Cezary Ziółkowski and Jan M. Kelner \\ Institute of Telecommunications, Faculty of Electronics, Military University of Technology, Gen. Sylwestra Kaliskiego Street No. 2, \\ 00-908 Warsaw, Poland \\ Correspondence should be addressed to Jan M. Kelner; jan.kelner@wat.edu.pl
}

Received 25 September 2015; Accepted 25 November 2015

Academic Editor: Ana Alejos

Copyright (C) 2015 C. Ziółkowski and J. M. Kelner. This is an open access article distributed under the Creative Commons Attribution License, which permits unrestricted use, distribution, and reproduction in any medium, provided the original work is properly cited.

\begin{abstract}
The paper presents an estimation of the reception angle distribution based on temporal characteristics such as the power delay spectrum (PDS) or power delay profile (PDP). Here, we focus on such wireless environment, where the propagation phenomenon predominates in azimuth plane. As a basis to determine probability density function (PDF) of the angle of arrival (AOA), a geometrical channel model (GCM) in form of the multielliptical model for delayed scattering components and the von Mises' PDF for local scattering components are used. Therefore, this estimator is called the distribution based on multielliptical model (DBMM). The parameters of GCM are defined on the basis of the PDS or PDP and the relative position of the transmitter and the receiver. In contrast to the previously known statistical models, DBMM ensures the estimation PDF of AOA by using the temporal characteristics of the channel for differing propagation conditions. Based on the results of measurements taken from the literature, DBMM verification, assessment of accuracy, and comparison with other models are shown. The results of comparison show that DBMM is the only model that provides the smallest least-squares error for different environments.
\end{abstract}

\section{Introduction}

In wireless communications, an angle distribution of the radio wave multipath components significantly affects the statistical properties of the received signals. Numerous measurements show that the environmental properties have a significant effect on the angle of arrival (AOA) of the receiving radio wave. Therefore, to perform statistical modeling of the impact of the propagation environment on deformations in time-spectrum of transmitted signals, knowledge of the probabilistic characteristics of angle is required. The search for new ways of transmitting information in wireless systems is primarily based on the results of simulation studies. Therefore, these characteristics are particularly important in these studies because their use provides the mapping of the effect of the object movement direction on the received signal.

One of the main ways of reception angle modeling is approximation of the measured data using standard probability density functions (PDFs). In this case, we talk about empirical models of angle distributions. In practice, Laplacian, Gaussian functions and Aulin's, Parson's models are the most commonly used distributions for azimuth [1] and elevation [2,3] angle, respectively. Unfortunately, there is no relationship that explicitly associates the parameters of these distributions with different types of propagation environments and the distance between the transmitter ( $\mathrm{Tx}$ ) and the receiver $(\mathrm{Rx})$. Therefore, the use of geometric channel models (GCMs) gives the possibility of taking into account the impact of changes in the position of the objects $(\mathrm{Tx}, \mathrm{Rx})$ on the spatial properties of the received signals. These models reproduce the geometry of the spatial relationships among $\mathrm{Tx}, \mathrm{Rx}$, and the location scattering areas. The shape of the scattering areas and spatial density of the scatterers are the criteria that differentiate the individual models. In literature, models of PDF of AOA are based primarily on such scatterer regions as circle [4-6], ellipse [5-7], elliptical disc [8], hollowdisc $[9,10]$, semispheroid $[4,11]$, clipping semispheroid [12], ellipsoid [13], and bounded ellipsoid [14] for two-dimensional 
(2D) and three-dimensional (3D) models, respectively. To the description of the spatial density of scatterers, such distributions are used as 2D uniform $[4,5,9,15,16], 3 \mathrm{D}$ uniform [4, 12], 2D Gaussian [17, 18], 3D Gaussian [12], hyperbolic [19], Rayleigh and exponential [20], parabolic $[6,14]$, "inverted parabolic" [21], and conical [22]. GCMs provide the basis for theoretical analysis of PDFs of AOA. Each of these models is described by one, two, or more parameters. However, it is difficult to identify the physical premises that bind the values of these parameters with the propagation properties of environment. Therefore, there is no clear relationship between the PDF model parameters and the propagation conditions. For this reason, the verification of these PDFs in the literature is reduced to a measurement data approximation only. In this paper, the method of determining the structure of GCM is significantly different from existing solutions. In our proposition, the parameters of the temporal characteristics of channel are the basis for GCM. As a result, statistical model of AOA is PDF estimator closely related to the type of propagation environment.

In most land mobile access systems, the half power beamwidth (HPBW) of antenna pattern is $360^{\circ}$ (omnidirectional antenna), or at least several tens of degrees in the azimuth plane. In elevation plane, HPBW has a few or at most a dozen degrees only. In this case, the phenomena modeling in the azimuth determines the mapping quality of the real signal. Therefore, these modeling conditions are decisive in simulation studies of wireless mobile channel. Unfortunately, none of the existing models does ensure the accuracy of the analytical description of the actual statistical properties of the AOA for different environmental conditions. Confirmed with the correctness of these observations are the results of a comparative analysis of the PDF of AOA for azimuth models that are presented in [23]. This paper presents an evaluation of the accuracy of the mapping format of azimuth AOA by various $2 \mathrm{D}$ GCMs for different propagation scenarios. The results for selected measuring scenarios are reference data, whereas the accuracy measure of the models is the least-square error (LSE). The summary of [23] is that "...no one geometric model is best by all criteria and for all environments...." As an exception to this conclusion, we present an analytical model, estimator of the azimuth AOA distribution for multipath homogeneous environments. This PDF estimator is based on temporal characteristics of the channel such as the power delay spectrum (PDS) or power delay profile (PDP). For a given type of environment, the parameters of these characteristics for different positions of $\mathrm{Tx} / \mathrm{Rx}$ are the estimation parameters.

The developed PDF estimator is based on the ParsonBajwa multielliptical GCM [19]. The geometry of this model results from the PDS or PDP. Literature analysis of the measurement results justifies such a methodology for determining PDF estimator. In [1], the correlation coefficient between delay and azimuth angle parameters is computed on the basis of the measurement data. Obtained linear regression line shows that these parameters are highly correlated [1, Figure 8]. Therefore, the developed model ensures a clear reproduction of the angle statistical properties with respect to temporal characteristic that is associated with the type of environment. In simulation studies, this fact makes it possible to adopt the model to the specific environment that is described by temporal characteristics of radio channel.

The purpose of this study is to show that the developed PDF model of azimuth AOA for different environments provides the minimum value of the estimation error in comparison to the models previously presented in the literature. Our work concerns the uniform environments, which means that the comparative analysis is focused on the propagation scenarios with unimodal PDF of azimuth angle.

The paper is organized as follows. Section 2 presents the analysis principles of reception angle distribution for the developed model. Section 3 describes in detail AOA analysis for delayed components as well as methodology of von Mises' distribution application for local scattering components. Methodology of adaptation of model parameters is presented in Section 4. This adaptation is based on the channel characteristics and the parameters of measurement scenario. Based on the results of measurements taken from the literature, model verification, assessment of accuracy, and comparison with other PDFs of AOA models are shown in Section 5. Conclusion, which highlights the practical use of the model to predict the angle distribution of received signal at different environments, is presented in Section 6.

\section{Distribution of Reception Angle: Principles of Analysis}

As shown by numerous measurement data, for example, [2426], due to multipath propagation of environment, a few to several replicas of the transmitted signal are received with various powers and time delays. This means that the received signal consists of the components. These components make up the time-clusters with a specific time delay, $\tau_{i}(i=1, \ldots, N$ is number of clusters), relative to the direct path.

The directivity, polarization, and gain from antenna patterns that are practical applications in wireless mobile communications are the reason for the dominance of the signal components, which are concentrated in the azimuth plane. It follows that the statistical properties of the received angle can be limited to the angle $\beta$ in the azimuth plane. These comments indicate that the angular distribution of power can be represented as power azimuth spectrum (PAS), $P(\beta)$, in the form

$$
P(\beta)=\sum_{i=1}^{N} P_{i}(\beta)+P_{0}(\beta),
$$

where $P_{i}(\beta)$ is PAS of the $i$ th time-cluster and $P_{0}(\beta)$ is PAS for local scatterings, that is, for cluster with negligibly small delay relative to the direct path.

However, the angle distribution and intensity of the direct path signal change with the change of $\mathrm{Tx} / \mathrm{Rx}$ position. In this case, PDF of AOA, $f(\beta)$, provides a basis for evaluation of the angular power dissipation and the Rice $\kappa$-factor describes the power distribution on direct path component and the 
components of local scatterings. The statistical description of PAS can be presented as [27]

$$
\begin{aligned}
P(\beta) & =P \cdot f_{\beta}(\beta) \\
& =\sum_{i=1}^{N} P_{i} f_{\beta i}(\beta)+\frac{1}{\kappa+1} P_{0} f_{0}(\beta)+\frac{\kappa}{\kappa+1} P_{0} \delta(\beta),
\end{aligned}
$$

where $P$ is the average power of the received signal, $P_{i}$ is the power of all components delay by $\tau_{i}$ (the $i$ th cluster), $f_{\beta i}(\beta)$ is PDF of AOA of multipath components delay by $\tau_{i}, P_{0}$ is the power of all components delay by zero, $f_{0}(\beta)$ is PDF of AOA of multipath components from local scatterings (delay by zero), and $\delta(\cdot)$ is the delta distribution.

Considering that $P=\int_{-\pi}^{\pi} P(\beta) \mathrm{d} \beta=\sum_{i=0}^{N} P_{i}$, we have

$$
\begin{aligned}
f_{\beta}(\beta)= & \sum_{i=1}^{N} p_{i} f_{\beta i}(\beta)+\frac{1}{\kappa+1} p_{0} f_{0}(\beta) \\
& +\frac{\kappa}{\kappa+1} p_{0} \delta(\beta),
\end{aligned}
$$

where $p_{i}=P_{i} / P$ is the normalized power of the $i$ th timecluster.

Equation (3) shows that the statistical properties of the signal reception angle are determined by the components arriving with a delay to $\mathrm{Rx}$ and components from local scatterings. For delayed components, an analysis of reception angle is based on multielliptical channel model, whereas, for local scattering components, von Mises' PDF is used. Thus, the analytical form of PDF of AOA is called the distribution based on multielliptical model (DBMM). The basis for the DBMM parameters can be characteristics such as power delay profile (PDP) or power delay spectrum (PDS) that determine $p_{i}$ values and the size of each ellipse. An additional input parameter is the height of the receiving antenna relative to the height of surrounding objects. This parameter is the basis for the assessment of local scattering intensity and determines the value of von Mises' PDF parameter. An analysis of the statistical properties of reception angle refers to homogeneous propagation environments. This means that such environments are dealt with for which the probability of a scatterer occurrence is the same for each direction when viewed from Tx. In practice, this means that the analysis covers those propagation scenarios that are characterized by unimodal PDF of the reception angle. In addition to the above considerations, to simplify the analyzed issues, the general assumptions that constitute the basis for most of GCMs are used $[12,16,17]$ :

(1) The radiation characteristics of the transmitting and receiving antennas are omnidirectional.

(2) Each propagation path from $\mathrm{Tx}$ to $\mathrm{Rx}$ consists of scatters on exactly one scattering element.

(3) For individual ellipses, each scatterer is a reradiating omnidirectional element with the same probability properties of scattering coefficient and uniform phase distribution.

\section{Statistical Model of Angle of Arrival}

The purpose of this work is to determine the analytical form of PDF of AOA of radio waves for a homogeneous multipath environment. The presented analysis uses the geometric mapping and statistical description of propagation phenomena. Propagation of delayed components is mapped by multielliptical GCM, while PDF of AOA of local scattering components is described by von Mises' PDF. In [27], a similar method of modeling the effects of propagation phenomena is used in analysis of the power azimuth spectrum (PAS).

The empirical studies demonstrate that, in time domain, the signal structure at the output of channels is shaped by a few or several clusters of the multipath components. These time-clusters are differentiated according to the values of power and time delay, after which the components arrive to $\mathrm{Rx}$. This means that, in the azimuth plane, the location of scatterers defines an ellipse for components that make up a single time-cluster. This fact is the basis for the use of the Parson and Bajwa model in the statistical analysis of AOA. In this case, GCM creates a set of $N$ ellipses that represent time-clusters with various $\tau_{i}$. The foci are common to all the ellipses. Their location determines the position of Tx and Rx, which are located at a distance $D$. Signal components, for which the arrival delay is negligibly small and is expressed only in the phase difference relative to the direct component, form a particular time-cluster. These components are the result of local scattering that occurs in proximity to $\mathrm{Tx} / \mathrm{Rx}$ antenna. The above model reproduces statistical location of the scatterers and thus provides the basis for determining PDF of AOA. The geometric structure of the considered problem and the adopted notations on the example of a chosen ellipse are shown in Figure 1. In addition, the local scattering area is highlighted in this figure.

The selected scatterer on a coordinate $x$ is marked as $S$. The angle of departure (AOD) from Tx and AOA at Rx are denoted by $\alpha$ and $\beta$, respectively. For each ellipse, the major axis $2 a_{i}$ depends on both $D$ and $\tau_{i}$ relative to direct path; namely,

$$
2 a_{i}=r_{1 i}+r_{2 i}=D+c \tau_{i},
$$

where $r_{1 i}$ is the path length between Tx and $S, r_{2 i}$ is the path length between $S$ and $\mathrm{Rx}$, and $c$ is the speed of light.

3.1. Statistical Properties of AOA for Delay Components. In this section, for individual PDF components, a brief analysis from [27] is presented. Based on the geometric properties of an ellipse and taking into account coordinate system as in Figure 1, it results that

$$
\begin{aligned}
& r_{1 i}=a_{i}-e_{i} x, \\
& r_{2 i}=a_{i}+e_{i} x,
\end{aligned}
$$

where $e_{i}=0.5 D / a_{i}<1$ is the eccentricity of the $i$ th ellipse. The coordinate $x$ can be expressed by $\alpha$ and $\beta$ as follows:

$$
\begin{aligned}
& x=r_{1 i} \cos \alpha+0.5 D, \\
& x=r_{2 i} \cos \beta-0.5 D .
\end{aligned}
$$




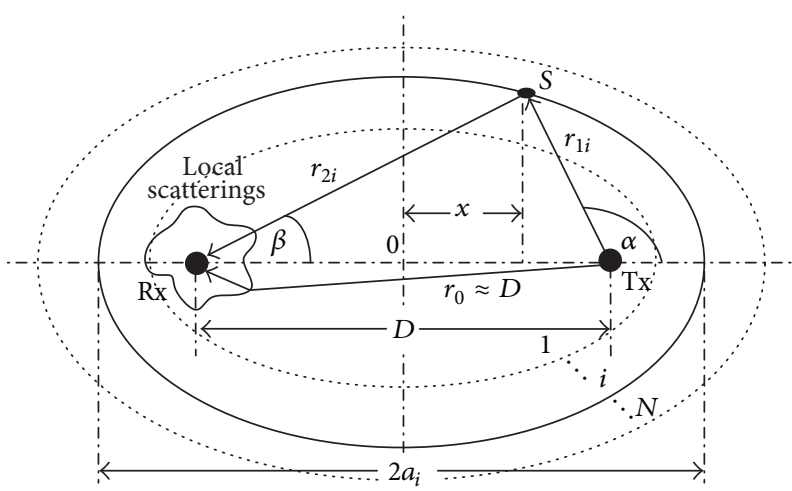

FIGURE 1: Geometry of the analyzed problem.

On the basis of relations (4)-(6), we get

$$
\frac{1}{1+e_{i} \cos \alpha}+\frac{1}{1-e_{i} \cos \beta}=\frac{2 a_{i}}{a_{i}-0.5 D} .
$$

By transforming the above expression, we can express $\cos \alpha$ as

$$
\cos \alpha=\frac{a_{i} \cos \beta+0.5 D e_{i} \cos \beta-D}{a_{i}+0.5 D e_{i}-D \cos \beta} .
$$

$$
\begin{aligned}
f_{c \beta}(\cos \beta) & =f_{c \alpha}(\cos \beta)\left|\frac{\mathrm{d}(\cos \alpha)}{\mathrm{d}(\cos \beta)}\right| \\
& =\frac{1}{2 \pi} \frac{1}{\sqrt{1-\left(\left(a_{i} \cos \beta+0.5 D e_{i} \cos \beta-D\right) /\left(a_{i}+0.5 D e_{i}-D \cos \beta\right)\right)^{2}}} \frac{\left(a_{i}+0.5 D e_{i}\right)^{2}-D^{2}}{\left(a_{i}+0.5 D e_{i}-D \cos \beta\right)^{2}} \\
& =\frac{1}{2 \pi} \frac{1}{\sqrt{1-\cos ^{2} \beta}} \frac{\sqrt{\left(a_{i}+0.5 D e_{i}\right)^{2}-D^{2}}}{a_{i}+0.5 D e_{i}-D \cos \beta} .
\end{aligned}
$$

Let us note that

$$
\begin{aligned}
\left|\frac{\mathrm{d}(\cos \alpha)}{\mathrm{d}(\cos \beta)}\right| & =\left|\frac{\left(a_{i}+0.5 D e_{i}\right)^{2}-D^{2}}{\left(a_{i}+0.5 D e_{i}-D \cos \beta\right)^{2}}\right| \\
& =\frac{\left(a_{i}+0.5 D e_{i}\right)^{2}-D^{2}}{\left(a_{i}+0.5 D e_{i}-D \cos \beta\right)^{2}} .
\end{aligned}
$$

Thus, PDF of $\cos \beta$ can be expressed as
Therefore, for paths arriving at $\operatorname{Rx}$ with $\tau_{i}, \operatorname{PDF}$ of $\beta$ is

$$
\begin{aligned}
f_{i \beta}(\beta) & =f_{c \beta}(\cos \beta)\left|\frac{\mathrm{d}(\cos \beta)}{\mathrm{d} \beta}\right| \\
& =\frac{1}{2 \pi} \frac{\sqrt{\left(a_{i}+0.5 D e_{i}\right)^{2}-D^{2}}}{a_{i}+0.5 D e_{i}-D \cos \beta},
\end{aligned}
$$

but

$$
\begin{aligned}
& \sqrt{\left(a_{i}+0.5 D e_{i}\right)^{2}-D^{2}}=a_{i} \sqrt{\left(1+e_{i}^{2}\right)^{2}-\left(2 e_{i}\right)^{2}} \\
& =a_{i} \sqrt{\left(1+e_{i}^{2}+2 e_{i}\right)\left(1+e_{i}^{2}-2 e_{i}\right)} \\
& =a_{i} \sqrt{\left(1+e_{i}\right)^{2}\left(1-e_{i}\right)^{2}}=a_{i}\left(1+e_{i}\right)\left(1-e_{i}\right) \\
& =a_{i}\left(1-e_{i}^{2}\right),
\end{aligned}
$$

so, we have

$$
f_{i \beta}(\beta)=\frac{1}{2 \pi} \frac{1-e_{i}^{2}}{1+e_{i}^{2}-2 e_{i} \cos \beta}, \quad \beta \in\langle-\pi, \pi\rangle .
$$

The definition of the ellipse eccentric indicates that $f_{i \beta}(\beta)$ depends on $D$ and $\tau_{i}$. The first parameter describes the geometry of propagation scenario, and the second is clear from propagation properties of the environment.

3.2. Statistical Properties of AOA for Local Scattering Components. Uniform distribution is one of the basic models describing statistical properties of AOA for the local scattering components. This distribution is used to describe $f_{0}(\beta)$ in Clarke's 2D isotropic scattering model [20]. Examples of other distributions related to the local scatterings in the 
surroundings of the receiving antenna are presented in $[15$, 18]. As shown in [18], the von Mises PDF expressed by

$$
f_{0}(\beta)=\frac{\exp (\mu \cos \beta)}{2 \pi I_{0}(\mu)}, \quad \beta \in\langle-\pi, \pi\rangle, \mu \geq 0,
$$

where $I_{0}(\cdot)$ is the zero-order modified Bessel function and $\mu$ is a constant that controls the width of AOA of the scattering components, is a generalized distribution.

For $\mu=0$, we obtain $f_{i \beta}(\beta)=(2 \pi)^{-1}$ while $\mu \rightarrow \infty$ yields $f_{0}(\beta) \rightarrow \delta(\beta)$. This means that, with the increase in the scattering intensity of the propagation paths, the parameter value decreases. For small $\mu$, this function approximates the cardioid while for large values it resembles the Gaussian PDF. In practice, $\mu$ depends on the height and the density of objects in the surrounding of the receiving antenna. In [18], the presented measurement results show that if the antenna height is less than the height of the surrounding objects, then $\mu \leq 3.3$.

3.3. Analytical PDF of AOA Model. The dependences (3), (14), and (15) are the basis for the determination of the analytical form of PDF of AOA

$$
\begin{aligned}
f_{\beta}(\beta)= & \frac{1}{2 \pi} \sum_{i=1}^{N} p_{i} \frac{1-e_{i}^{2}}{1+e_{i}^{2}-2 e_{i} \cos \beta} \\
& +p_{0} \frac{1}{\kappa+1} \frac{\exp (\mu \cos \beta)}{2 \pi I_{0}(\mu)}+p_{0} \frac{\kappa}{\kappa+1} \delta(\beta) .
\end{aligned}
$$

The above relation shows that $f_{\beta}(\beta)$ is significantly dependent on $e_{i}, p_{i}, \kappa$, and $\mu$. This means that the analyzed function depends on the distance between $\mathrm{Tx}$ and $\mathrm{Rx}$, the type of the environment, and the nature of the local scatterings. This means that $f_{\beta}(\beta)$ changes with change of the position of $\mathrm{Tx} / \mathrm{Rx}$. Thus, for a given type of propagation environment, (16) describes PDF estimator of AOA, whose parameters are determined on the basis of changing temporal characteristics of channel. Also note that (16) is theoretical due to the presence of $\delta$-distribution that describes PDF of $\mathrm{AOA}$ of the direct path. In practice, obtaining a result in the form of the distribution is not impossible. The reason for this is the limited measurement discreteness of angle that results from the limited width of the receiving antenna beam. Therefore, to evaluate the model approximation error with respect to measurement results, the distribution component should be replaced by a function that belongs to a set of delta-forming functions. These functions take nonzero values only for the interval that corresponds to the measurement discreteness of the angle, and their surface areas are equal to the normalized received signal power.

\section{Example of Numerical Results}

In this section, the methodology for determining PDF of AOA is described for selected test scenarios that the detailed characterization is presented in [1]. For DBMM, the input data are identified on the basis of the measurement of PDS that is shown in the graph in this paper ([1, Figure 6]).

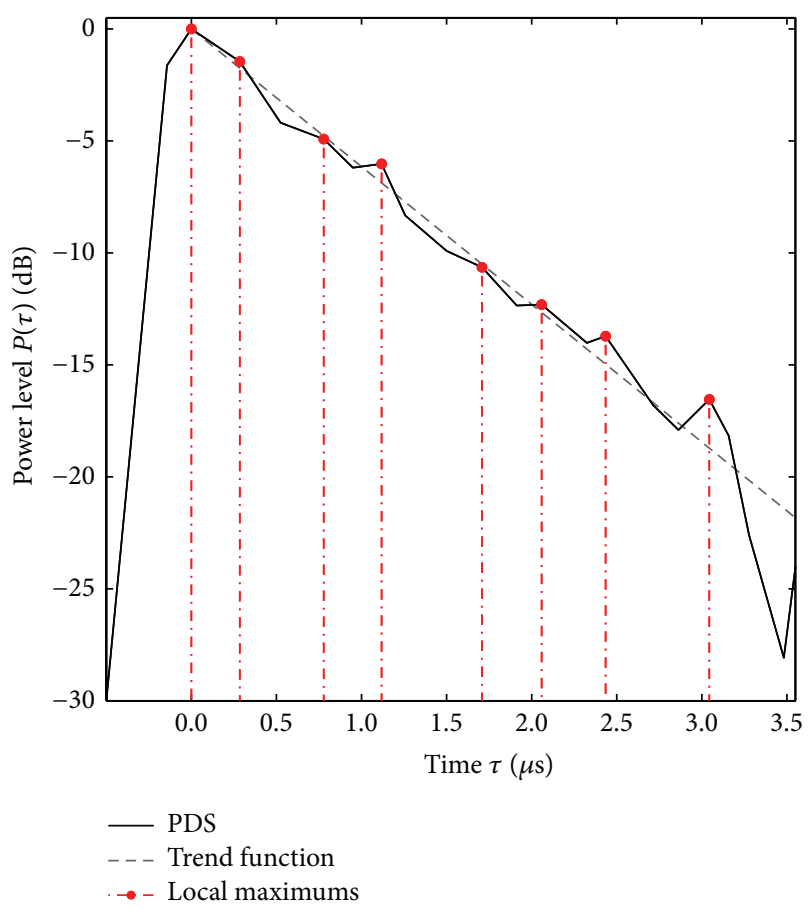

FIGURE 2: Identification of parameters for individual clusters on the example of measurement scenario from [1] (Stockholm).

Number of the time-clusters and the values of their parameters, that is, $N, \tau_{i}$, and $p_{i}$, are defined on the base of PDS selected points. These points are local extremes of the function, which is the difference between PDS and its trend. For the analyzed scenario, the function of trend is Laplacian function. The choice of points that identify the parameters of DBMM is shown in Figure 2, whereas the determined values are contained in Table 1.

The distance $D=1500 \mathrm{~m}$ between $\mathrm{Tx}$ and $\mathrm{Rx}$ is adopted based on the description of the scenario and analysis of measurement results presented in [4]. To assure consistence in extracting numerical data from graphs the software WebPlotDigitizer [31] is used. The dataset, contained in Table 1, defined a first group of components that are presented in expression (16). This group describes the angular power dispersion of the signal components that arrive at the receiver with delays. Parameters of remaining components of $f_{\beta}(\beta)$, that is, $\kappa$ and $\mu$, are determined by the propagation environment nature and parameters of the measuring system.

In [1], the described measurement campaign was conducted in urban area characterized by buildings ranging from four to six floors and placed on a slightly rolling terrain. The transmitting equipment is mounted in car. The transmitting antenna is omnidirectional whereas $\mathrm{Rx}$ is equipped with an eight-element uniform linear antenna array whose height corresponds to the average rooftop level, that is, $21 \mathrm{~m}$ above ground level. Thus, for the analyzed scenario, only the objects in immediate surroundings of $\mathrm{Tx}$ and the surfaces of roofs at the location of the receiving antenna are a source of local scatterings. This means that the local scatterings are targeted. In this case, it is the basis for the adoption of high-value of 
TABLE 1: Parameters for individual clusters $(N=7)$.

\begin{tabular}{lcccccc}
\hline $\begin{array}{l}\text { Cluster } \\
\text { number } \\
i\end{array}$ & $P_{i}(\mathrm{~dB})$ & $P_{i}$ & $p_{i}$ & $\tau_{i}(\mu \mathrm{s})$ & $a_{i}(\mathrm{~km})$ & $e_{i}$ \\
\hline 0 & 0.00 & 1.0000 & $\mathbf{0 . 4 0 0 3}$ & $\mathbf{0 . 0 0}$ & - & - \\
1 & -1.45 & 0.7161 & $\mathbf{0 . 2 8 6 7}$ & $\mathbf{0 . 2 8}$ & 0.7920 & 0.9470 \\
2 & -4.91 & 0.3228 & $\mathbf{0 . 1 2 9 2}$ & $\mathbf{0 . 7 8}$ & 0.8670 & 0.8651 \\
3 & -6.03 & 0.2495 & $\mathbf{0 . 0 9 9 9}$ & $\mathbf{1 . 1 2}$ & 0.9180 & 0.8170 \\
4 & -10.64 & 0.0863 & $\mathbf{0 . 0 3 4 5}$ & $\mathbf{1 . 7 1}$ & 1.0065 & 0.7452 \\
5 & -12.31 & 0.0587 & $\mathbf{0 . 0 2 3 5}$ & $\mathbf{2 . 0 6}$ & 1.0590 & 0.7082 \\
6 & -13.72 & 0.0425 & $\mathbf{0 . 0 1 7 0}$ & $\mathbf{2 . 4 3}$ & 1.1145 & 0.6729 \\
7 & -16.54 & 0.0222 & $\mathbf{0 . 0 0 8 9}$ & $\mathbf{3 . 0 4}$ & 1.2060 & 0.6219 \\
\hline
\end{tabular}

$\mu$ equal to 60 . Because the measurement was carried out with non-line-of-sight (NLOS) between $\mathrm{Tx}$ and $\mathrm{Rx}$, we have $\kappa=0$. Taking into account the above considerations, $f_{\beta}(\beta)$ reduces to the form

$$
\begin{aligned}
f_{\beta}(\beta)= & \frac{1}{2 \pi} \sum_{i=1}^{L} p_{i} \frac{1-e_{i}^{2}}{1+e_{i}^{2}-2 e_{i} \cos \beta} \\
& +p_{0} \frac{\exp (\mu \cos \beta)}{2 \pi I_{0}(\mu)},
\end{aligned}
$$

where $e_{i}$ and $p_{i}$ values are contained in Table 1 .

In Figure 3, comparison of the empirical data with the theoretical analysis results that are obtained on the base of (17) is presented.

As a measure of the accuracy of measurement data by DBMM, the Kolmogorov-Smirnov statistic, $D_{m}$, is used [32]:

$$
D_{m}=\sup _{\beta_{k}}\left|F_{m}\left(\beta_{k}\right)-F_{\beta}\left(\beta_{k}-\beta_{0}\right)\right|,
$$

where $F_{\beta}\left(\beta_{k}\right)=\int_{-\pi}^{\beta_{k}} f_{\beta}(\beta) \mathrm{d} \beta$ is the cumulative distribution function (CDF), $f_{\beta}\left(\beta_{k}\right)$ represents PDF of AOA for analyzed model, $F_{m}\left(\beta_{k}\right)=(1 / 2) \sum_{l=2}^{k}\left[f_{m}\left(\beta_{l-1}\right)+f_{m}\left(\beta_{l}\right)\right]\left(\beta_{l}-\beta_{l-1}\right)$ and $f_{m}\left(\beta_{k}\right)(k=1, \ldots, K)$ denote the normalized CDF and PDF from empirical dataset, respectively, $K$ refers to cardinality of the set of measurement data, and $\beta_{0}$ is a parameter that calibrates PDF of AOA model to the direction of Tx-Rx for empirical data. For analyzed scenario, we have $D_{m}=0.0264$.

In subsequent sections, $D_{m}$ is used to assess the accuracy of mapping the actual characteristics by the selected analytical models of PDF of AOA.

\section{Verification and Comparison of Models}

The main aim of this section is to verify the accuracy and compare DBMM with other statistical AOA models for which comprehensive comparative analysis is presented in [23]. According to the accepted assumptions in Section 2, comparative analysis refers to selected GCMs and measurement scenarios for which PDF of AOA is unimodal. On the base of the analysis results that are shown in [23] (Table 4), the comparison is reduced to a set of models that are best

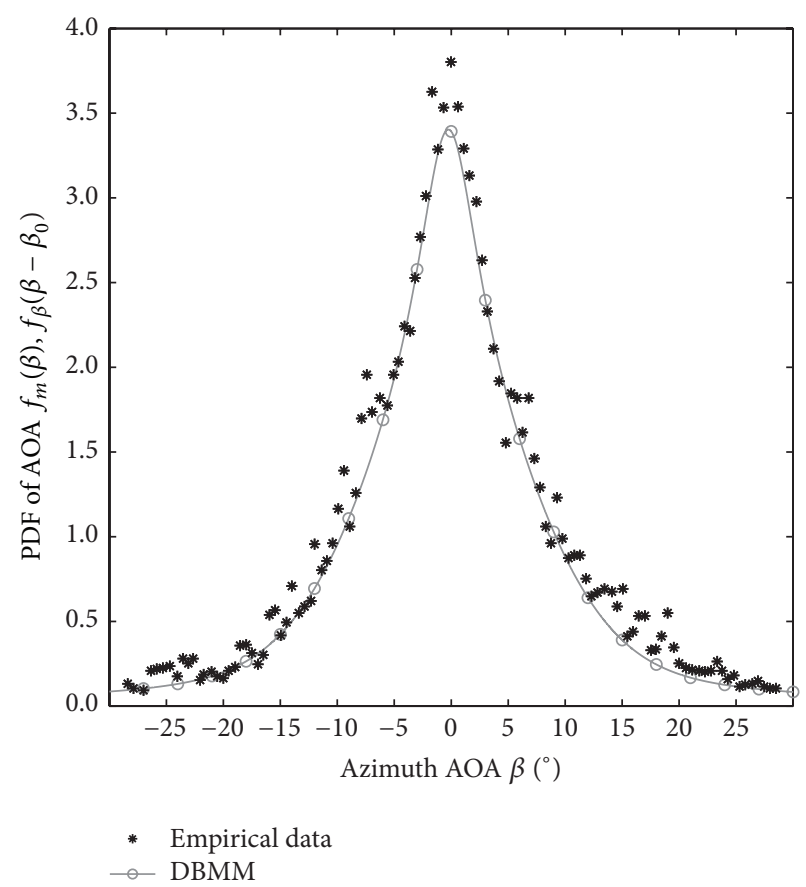

FIgURE 3: Comparing theoretical analysis to the empirical results in Pedersen et al. [1, Figure 4] (Stockholm).

matched to the empirical data. Table 2 shows the selected test scenarios and corresponding models that provide the best fit to the measurement results, whereas Table 3 contains the analytical dependences that define PDFs of AOA for different GCMs based on [23]. Additionally, in accordance with the suggestion made in the conclusion [23], the Gaussian model is included in comparison analysis.

In [23], LSE is used as an objective function with the minimizing criterion for determining the parameters of the analyzed models:

$$
\mathrm{LSE}=\frac{1}{K} \sum_{k=1}^{K}\left(f_{m}\left(\beta_{k}\right)-f_{\beta}\left(\beta_{k}-\beta_{0}\right)\right)^{2} .
$$

The parameters of DBMM are determined otherwise. The bases for the determination of $N, \tau_{i}$, and $p_{i}$ are the temporal channel characteristics such as PDS or PDP. For each scenario, the empirical data is not a uniform set. For $[1,28,33]$ scenarios, PDS is the basis for DBMM parameters, so all of them take statistically constant values. PDP is used with respect to $[26,29,30,34]$ scenarios. In these cases, (16) represents PDF estimator of AOA that is associated with the specific geometry of environment. The geometry of $\mathrm{Rx}$ antenna immediate surroundings determines the values of the other two parameters $(\kappa, \mu)$. Due to the lack of data, the ranges of these parameters are adopted on the basis of the description of the measurement conditions. Similarly, as in [23], the criterion of minimizing LSE is used to select $\kappa$ and $\mu$ values. In Section 4 , the methodology for determining the parameters of DBMM and then PDF of AOA is shown in the example of results obtained from the Stockholm 
TABLE 2: Selected test scenarios and the best matched models.

\begin{tabular}{lcc}
\hline \multicolumn{1}{c}{ Measurement scenario (empirical PDF of AOA) } & PDF of AOA models \\
\hline Matthews et al. [34, Figure 7] & (Wong et al. [23, Figure 4]) & $\begin{array}{l}\text { Gaussian } \\
\text { Rayleigh circular (Rx outside) }\end{array}$ \\
\hline Pedersen et al. [33, Figure 1] & (Wong et al. [23, Figure 5]) & Uniform elliptical (Rx outside) \\
\hline Kuchar et al. [28, Figure 3] & (Wong et al. [23, Figure 6]) & Gaussian \\
& & Rayleigh circular (Rx outside) \\
\hline Takada et al. [26, Figure 4] & (Wong et al. [23, Figure 7]) & Uniform elliptical (Rx outside) \\
\hline & & Uniform elliptical (Rx outside) \\
Fleury et al. [29, Figure 16] & (Wong et al. [23, Figure 8]) & Gaussian \\
\hline Mogensen et al. [30, Figure 3] & Rayleigh circular (Rx outside) \\
\hline Pedersen et al. [1, Figure 4], Aarhus & Uniform elliptical (Rx outside) \\
\hline Pedersen et al. [1, Figure 4], Stockholm & (Wong et al. [23, Figure 10]) & Uniform elliptical (Rx outside) \\
\hline
\end{tabular}

measurement scenario [1]. In the same way, this methodology is used for the second measurement scenario from [1] (Aarhus) and scenarios from [29, 34]. For measurement scenarios from Stockholm and Aarhus, different PDSs and the amount of the receiving antennas with respect to the environment objects are the causes of various parameters for DBMM. In measurement campaign that was conducted in Aarhus, the receiving antenna was mounted at higher altitude, that is, $12 \mathrm{~m}$, than average rooftop level of the surrounding buildings. Thus, the scattering that occurs in the vicinity of the transmitting antenna especially determines the local scattering. This is the reason for reducing the scope of these scatterings, which is a prerequisite for increasing $\mu$ from 60 for Stockholm to 120 for Aarhus.

Because of the nature of the propagation phenomena that are dominant in [26] measurement scenario, the methodology for determining PDF of AOA, on the basis of DBMM, is slightly modified. In this case, the amount of transmitting and receiving antennas was $2.7 \mathrm{~m}$ and $4.4 \mathrm{~m}$, respectively. Mutual location of Tx and Rx and the average height of the buildings, that is, $8 \mathrm{~m}$, to a significant extent determine the nature of the propagation environment. For this measurement scenario, an environment has the features of the canyon. This means that the propagation paths that run along the canyon have a dominant influence on the received signal. The geometry of the environment and the location of Tx show that the direct paths to the scattering places, located within the canyon, only concern the first three ellipses. Thus, for $\tau_{1}=0.02 \mu \mathrm{s}, \tau_{2}=$ $0.06 \mu \mathrm{s}$, and $\tau_{3}=0.08 \mu \mathrm{s}, f_{i \beta}(\beta)(i=1,2,3)$ are different from zero for $\beta \in\left\langle-2.25^{\circ}, 2.25^{\circ}\right\rangle$. Relative to the position of $\mathrm{Rx}$, this range represents the canyon angular width that determines the location of the scatterers. Geometrically, these places are represented by three subsequent sections of ellipses that are presented in the surroundings of Tx.

As presented in [30], the measurement scenario does not contain characteristics that describe the propagation properties of the environment. This makes it difficult to define the parameters of DBMM for the analyzed scenario. However, measurement campaigns that are described in [29, 30] relate to the same environment in Aalberg. Therefore, for these scenarios, the same parameter values of other models are adopted in [23]. Also, in [28, 33], the propagation properties of environments are not described. For these scenarios, DBMM parameters are defined on the basis of descriptions of the measurement conditions. They show that the measurement was carried out on frequencies of the standard GSM 1800 in rural and suburban environments for $[28,33]$, respectively. These facts are a premise for the use of parameter values that describe the rural environment in accordance with COST 207 model [35]. The descriptions of these scenarios also show that PAS measurements were conducted in line-of-sight (LOS) propagation conditions. The presence of the direct path determines AOA that theoretically is described by $p_{0}[\kappa /(\kappa+1)] \delta(\beta)$. In this case, comparative analysis of analytical dependence (16) and empirical results is difficult due to $\delta(\beta)$ presence. In practice, the minimum beam width of the measurement antenna pattern is the basis to replace $\delta(\beta)$ by a triangular function. This function belongs to the set of $\delta$-generating functions and maps the signal power arriving from the direction of the direct path. Hence, the span angle, $\Delta \phi$, for which the triangular function takes values different from zero, is associated with angular measurement discreteness that determines the beam width of the antenna pattern. Owing to the lack of accurate data, for measurement scenario [33], $\Delta \phi=2.5^{\circ}$ is adopted. In [28], the presented graphs are averaged measurement results that have been obtained over the entire measurement route. Therefore, in this case, $\Delta \phi$ is equal to the angular width of measurement route that is determined relative to the location of the receiving antenna. On the basis of the simplified scenario map, $\Delta \phi=15^{\circ}$ is adopted. In both cases, the surface area of triangular functions depends on the level of direct path signal power and amounts $p_{0} \kappa /(\kappa+1)$.

Distance between $\mathrm{Tx}$ and $\mathrm{Rx}$ is one of the important parameters that determine the geometry of the models. For $[26,34]$ scenarios, $D$ values are strictly defined, whereas, for other measurement campaigns, they are adopted as the average $\mathrm{Tx}$ distance relative to $\mathrm{Rx}$ location. The set of all DBMM parameters that are determined on the basis of descriptions of analyzed measurement scenarios is contained 


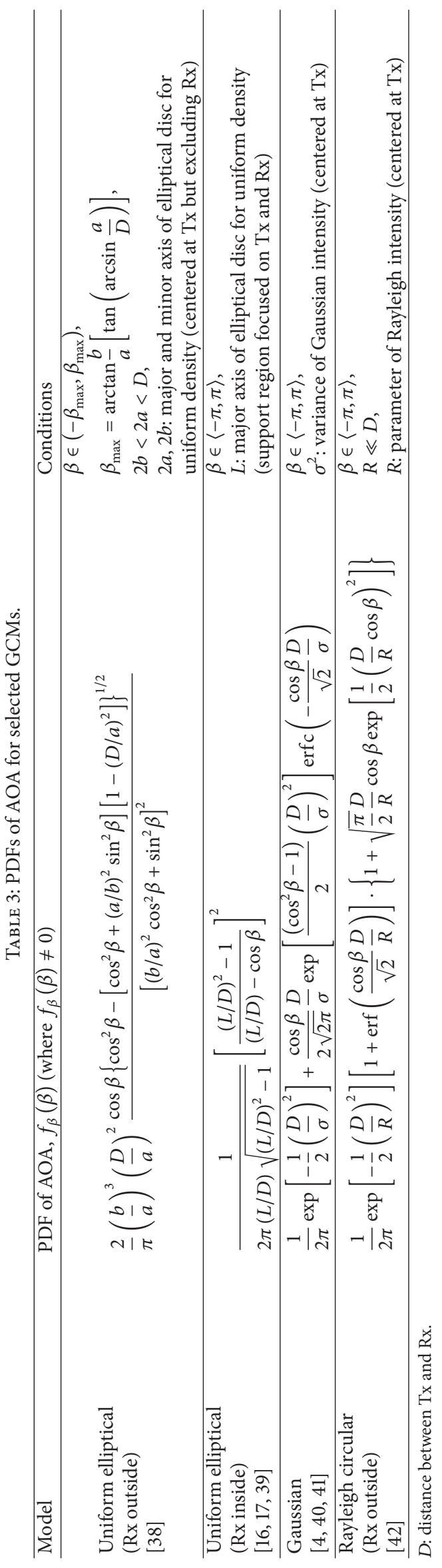


TABLE 4: Parameters of DBMM for analyzed measurement scenarios.

\begin{tabular}{|c|c|c|c|c|c|c|c|c|}
\hline $\begin{array}{l}\text { Measurement } \\
\text { scenario }\end{array}$ & {$[34]$} & [33] & {$[28]$} & {$[26]$} & [29] & {$[30]$} & $\begin{array}{c}\text { [1] } \\
\text { Aarhus }\end{array}$ & $\begin{array}{c}{[1]} \\
\text { Stockholm } \\
\end{array}$ \\
\hline $\begin{array}{l}\text { Location of } \\
\text { measurement }\end{array}$ & $\begin{array}{l}\text { Leeds, } \\
\text { England }\end{array}$ & $\begin{array}{l}\text { Bristol, } \\
\text { England }\end{array}$ & $\begin{array}{l}\text { Stuttgart, } \\
\text { Germany }\end{array}$ & $\begin{array}{c}\text { Yokosuka, } \\
\text { Japan }\end{array}$ & $\begin{array}{l}\text { Aalborg, } \\
\text { Denmark }\end{array}$ & $\begin{array}{l}\text { Aalborg, } \\
\text { Denmark }\end{array}$ & $\begin{array}{l}\text { Aarhus, } \\
\text { Denmark }\end{array}$ & $\begin{array}{c}\text { Stockholm, } \\
\text { Sweden }\end{array}$ \\
\hline$D(\mathrm{~m})$ & 1800 & 5000 & 400 & 219 & 2100 & 2100 & 1500 & 1500 \\
\hline$\mu$ & 60 & 240 & 90 & 200 & 140 & 140 & 120 & 60 \\
\hline$\kappa$ & 0 & 1 & 0.3 & 0 & 0 & 0 & 0 & 0 \\
\hline$\Delta \phi\left(^{\circ}\right)$ & - & 2.5 & 15 & - & - & - & - & - \\
\hline$\beta_{0}\left(^{\circ}\right)$ & 23.4 & 0 & 3.5 & 2.96 & 24.86 & 0.8 & 0.26 & 0.25 \\
\hline $\begin{array}{l}\text { PDP/PDS } \\
\text { based on }\end{array}$ & $\begin{array}{c}\text { [34, Figure } \\
10]\end{array}$ & $\begin{array}{l}\text { COST } 207 \\
{[35]} \\
\text { rural area }\end{array}$ & $\begin{array}{l}\text { COST } 207 \\
\quad[35] \\
\text { rural area } \\
\text { [modified] }\end{array}$ & [26, Figure 5] & $\begin{array}{c}{[29, \text { Figure }} \\
16]\end{array}$ & $\begin{array}{c}{[29, \text { Figure }} \\
16]\end{array}$ & {$[1$, Figure 6] } & {$[1$, Figure 6$]$} \\
\hline$N$ & 6 & 5 & 5 & 7 & 4 & 4 & 5 & 7 \\
\hline$p_{0}$ & 0.8506 & 0.6024 & 0.8584 & 0.2115 & 0.4735 & 0.4735 & 0.4363 & 0.4003 \\
\hline$p_{i}$ & $\begin{array}{l}0.1060 \\
0.0126 \\
0.0188 \\
0.0083 \\
0.0021 \\
0.0016\end{array}$ & $\begin{array}{c}0.2410 \\
0.0964 \\
0.0361,0.0181 \\
0.0060\end{array}$ & $\begin{array}{l}0.0858 \\
0.0343 \\
0.0129 \\
0.0064 \\
0.0022\end{array}$ & $\begin{array}{c}0.2061 \\
0.0814 \\
0.3178,0.1192 \\
0.0158 \\
0.0436 \\
0.0046\end{array}$ & $\begin{array}{l}0.4442 \\
0.0733 \\
0.0049 \\
0.0041\end{array}$ & $\begin{array}{l}0.4442 \\
0.0733 \\
0.0049 \\
0.0041\end{array}$ & $\begin{array}{l}0.3019 \\
0.2091 \\
0.0436 \\
0.0074 \\
0.0017\end{array}$ & $\begin{array}{l}0.2867 \\
0.1292 \\
0.0999 \\
0.0345 \\
0.0235 \\
0.0170 \\
0.0089 \\
\end{array}$ \\
\hline$\tau_{i}(\mu \mathrm{s})$ & $\begin{array}{l}01.22,01.86 \\
02.47,06.52 \\
07.73,10.84\end{array}$ & $\begin{array}{l}0.10,0.20 \\
0.30,0.40 \\
\quad 0.50\end{array}$ & $\begin{array}{l}0.10,0.20 \\
0.30,0.40 \\
\quad 0.50\end{array}$ & $\begin{array}{c}0.02,0.06 \\
0.08,0.14 \\
0.19,0.32 \\
0.48\end{array}$ & $\begin{array}{l}0.95,3.76 \\
5.61,8.36\end{array}$ & $\begin{array}{l}0.95,3.76 \\
5.61,8.36\end{array}$ & $\begin{array}{c}0.14,0.32 \\
0.83,1.28 \\
\quad 1.95\end{array}$ & $\begin{array}{c}0.28,0.78 \\
1.12,1.71,2.06 \\
2.43,3.04\end{array}$ \\
\hline
\end{tabular}

TABLE 5: Parameters of selected models that minimize LSE [23].

\begin{tabular}{|c|c|c|c|c|c|c|c|c|}
\hline $\begin{array}{l}\text { Measurement } \\
\text { scenario }\end{array}$ & {$[34]$} & {$[33]$} & {$[28]$} & {$[26]$} & [29] & {$[30]$} & $\begin{array}{c}{[1]} \\
\text { Aarhus }\end{array}$ & $\begin{array}{c}{[1]} \\
\text { Stockholm }\end{array}$ \\
\hline \multicolumn{9}{|c|}{$\begin{array}{l}\text { Uniform elliptical } \\
\text { (Rx outside) }\end{array}$} \\
\hline$b / a$ & 0.27 & 0.03 & 0.21 & 0.05 & 0.17 & 0.16 & 0.11 & 0.20 \\
\hline$a / D$ & 0.81 & 0.99 & 0.81 & 0.96 & 0.99 & 0.99 & 0.99 & 0.99 \\
\hline \multicolumn{9}{|c|}{$\begin{array}{l}\text { Uniform elliptical } \\
\text { (Rx inside) }\end{array}$} \\
\hline$L / D$ & 1.0204 & 1.0101 & 1.0101 & 1.0101 & 1.0101 & 1.0101 & 1.0101 & 1.0204 \\
\hline \multicolumn{9}{|l|}{ Gaussian } \\
\hline$\sigma / D$ & 0.14 & 0.02 & 0.11 & 0.03 & 0.10 & 0.10 & 0.07 & 0.13 \\
\hline \multicolumn{9}{|c|}{$\begin{array}{l}\text { Rayleigh circular } \\
\text { (Rx outside) }\end{array}$} \\
\hline$R / D$ & 0.139 & 0.027 & 0.110 & 0.032 & 0.104 & 0.098 & 0.073 & 0.134 \\
\hline
\end{tabular}

in Table 4. Based on [23], the values of parameters that minimize LSE for selected models are presented in Table 5.

Assessment of the accuracy of individual models is based on an assessment of $D_{m}(18)$ with respect to the measurement results for different environments. In Table 6, the results of numerical calculations are presented.

Additionally, the results obtained by Wong et al. [23] and the results for DBMM are shown in Table 7. Graphical illustrations of PDF of AOA models and empirical data for the analyzed scenarios are presented in Figures 4-11.
For different environments, the analysis of $D_{m}$ and LSE show that, among the analytical models of PDF of AOA, DBMM is the model that accurately reproduces the results of measurements for all analyzed scenarios. The characteristics of the received signals and environmental conditions are the basis for DBMM parameters listed in Table 4. As a result, DBMM is closely related to the properties of each environment, which results in minimizing $D_{m}$ and LSE in comparison with other models. Seemingly high degree of freedom that is in DBMM results from a larger number 
TABLE 6: Kolmogorov-Smirnov statistic for selected models calibrated by empirical data.

\begin{tabular}{|c|c|c|c|c|c|c|c|c|}
\hline Measurement scenario & {$[34]$} & {$[33]$} & {$[28]$} & {$[26]$} & [29] & {$[30]$} & $\begin{array}{c}{[1]} \\
\text { Aarhus }\end{array}$ & $\begin{array}{c}{[1]} \\
\text { Stockholm }\end{array}$ \\
\hline Uniform elliptical (Rx outside) & 0.0238 & 0.0654 & 0.0129 & 0.0733 & 0.0343 & 0.1001 & 0.0558 & 0.0309 \\
\hline Uniform elliptical (Rx inside) & 0.0306 & 0.1890 & 0.0384 & 0.1584 & 0.0636 & 0.1148 & 0.0883 & 0.0314 \\
\hline Gaussian & 0.0256 & 0.0909 & 0.0107 & 0.1017 & 0.0554 & 0.1004 & 0.0660 & 0.0429 \\
\hline Rayleigh circular (Rx outside) & 0.0239 & 0.0684 & 0.0107 & 0.0937 & 0.0492 & 0.1029 & 0.0630 & 0.0395 \\
\hline DBMM & 0.0196 & 0.0376 & 0.0083 & 0.0548 & 0.0271 & 0.0868 & 0.0473 & 0.0264 \\
\hline
\end{tabular}

TABLE 7: LSE for selected models calibrated by empirical data.

\begin{tabular}{lcccccccc}
\hline Measurement scenario & {$[34]$} & {$[3]$} & {$[28]$} & {$[26]$} & {$[29]$} & {$[30]$} & $\begin{array}{c}{[1]} \\
\text { Aarhus }\end{array}$ & $\begin{array}{c}{[1]} \\
\text { Stockholm }\end{array}$ \\
\hline Uniform elliptical (Rx outside) & 0.0054104 & $\mathbf{1 . 6 4 8 3}$ & 0.0069964 & $\mathbf{0 . 1 2 6 9 9}$ & $\mathbf{0 . 0 2 0 0 9 9}$ & $\mathbf{0 . 0 1 4 3 8 0}$ & $\mathbf{0 . 0 8 4 3 6 3}$ & $\mathbf{0 . 0 4 3 0 7 5}$ \\
Uniform elliptical (Rx inside) & 0.0098299 & 39.6960 & 0.0322370 & 1.15250 & 0.027642 & 0.018240 & 0.196290 & $\mathbf{0 . 0 4 0 0 8 8}$ \\
Gaussian & $\mathbf{0 . 0 0 4 1 7 1 9}$ & 3.2328 & $\mathbf{0 . 0 0 6 0 8 0 7}$ & 0.14807 & $\mathbf{0 . 0 1 9 7 2 2}$ & 0.019087 & 0.191920 & 0.083137 \\
Rayleigh circular (Rx outside) & $\mathbf{0 . 0 0 4 1 5 2 6}$ & 6.3259 & $\mathbf{0 . 0 0 6 0 8 0 7}$ & 0.18178 & $\mathbf{0 . 0 1 8 8 4 1}$ & 0.018867 & 0.187050 & 0.081898 \\
DBMM & $\mathbf{0 . 0 0 3 0 1 4 1}$ & $\mathbf{1 . 0 9 6 3}$ & $\mathbf{0 . 0 0 5 4 1 6 7}$ & $\mathbf{0 . 1 1 3 8 9}$ & $\mathbf{0 . 0 1 2 7 7 0}$ & $\mathbf{0 . 0 1 0 4 8 2}$ & $\mathbf{0 . 0 5 0 5 1 5}$ & $\mathbf{0 . 0 2 6 1 6 4}$ \\
\hline
\end{tabular}

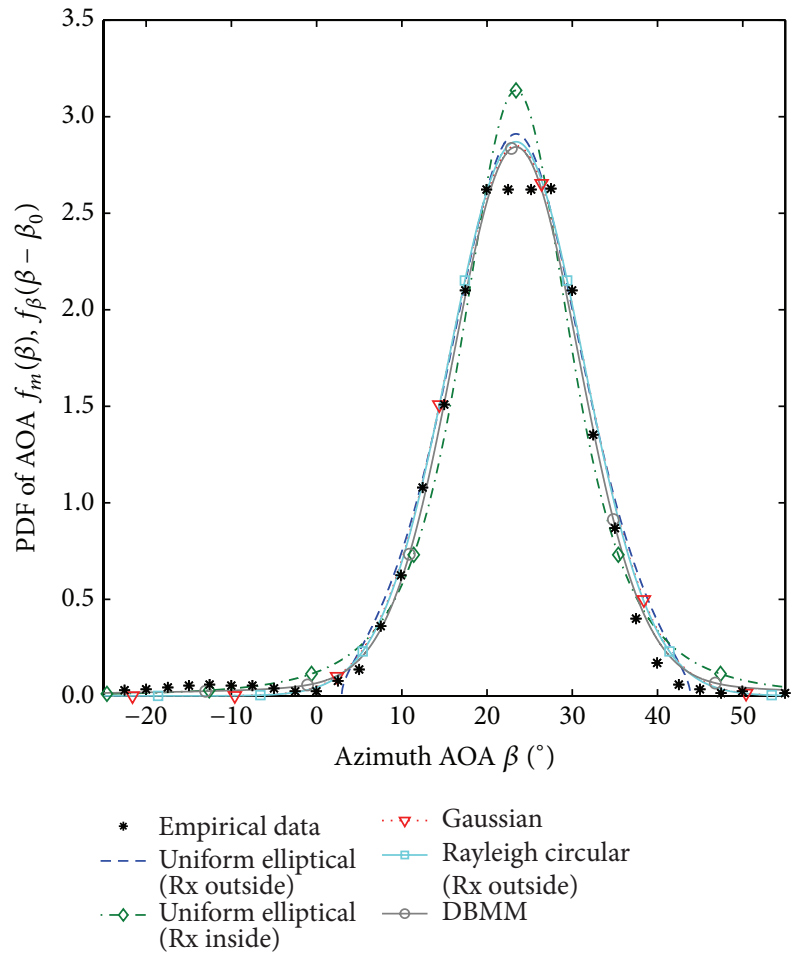

FIGURE 4: Fitting for various geometric models to the empirical data in Matthews et al. [34, Figure 7].

of the parameters. However, these parameters are closely determined based on the transmission conditions and the characteristics of the received signals.

For analytical models of PDF of AOA that are presented in [23], the parameters are practically immeasurable. This fact significantly impedes their use to predict the angle

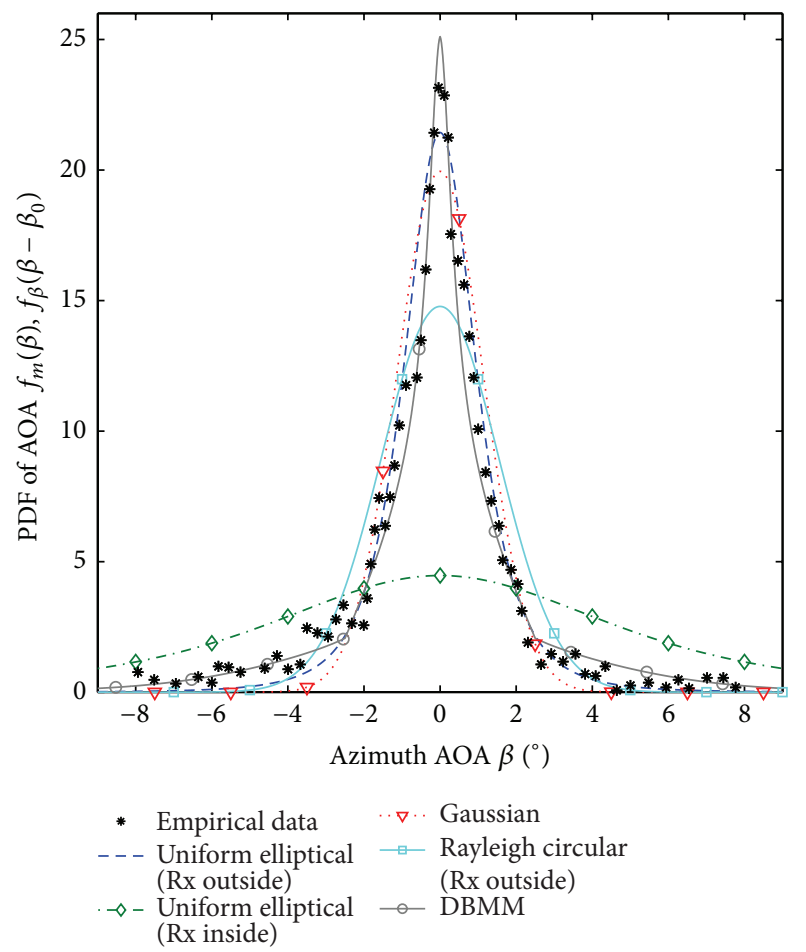

FIGURE 5: Fitting for various geometric models to the empirical data in Pedersen et al. [33, Figure 1].

distribution of received signals for different environments. In the case of DBMM, it is different. The characteristics (IR, PDP, or PDS) that are the basis for this model define the propagation properties of the environment. As a result, DBMM has the ability to predict PDF of AOA for environments with different propagation properties. 


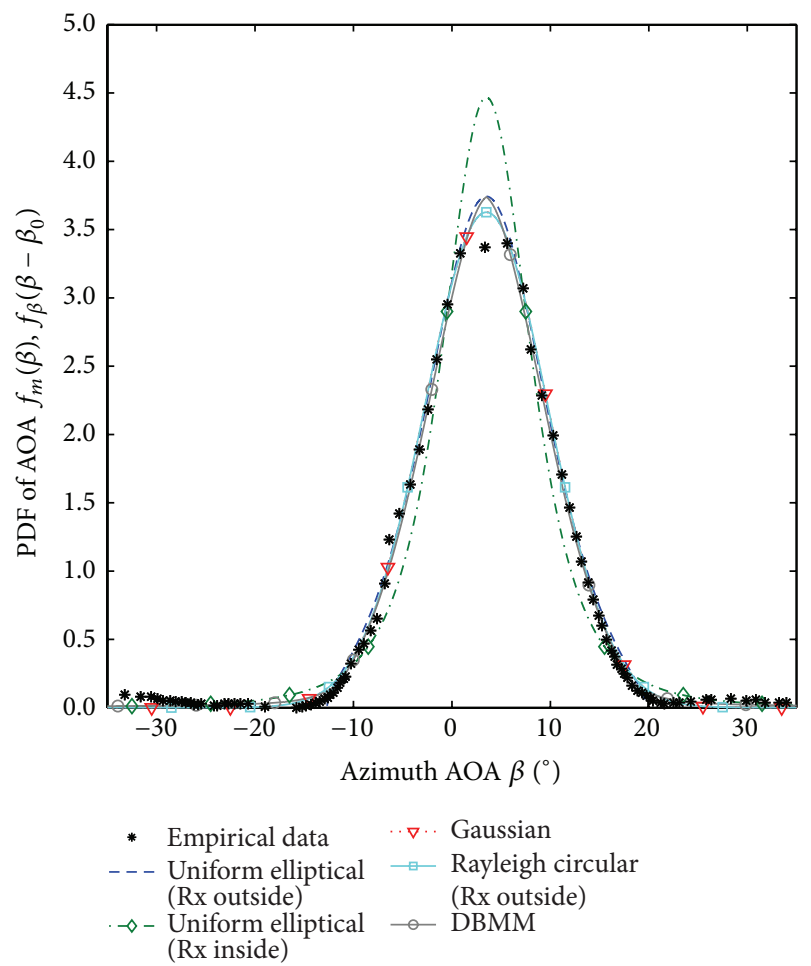

FIGURE 6: Fitting for various geometric models to the empirical data in Kuchar et al. [28, Figure 3].

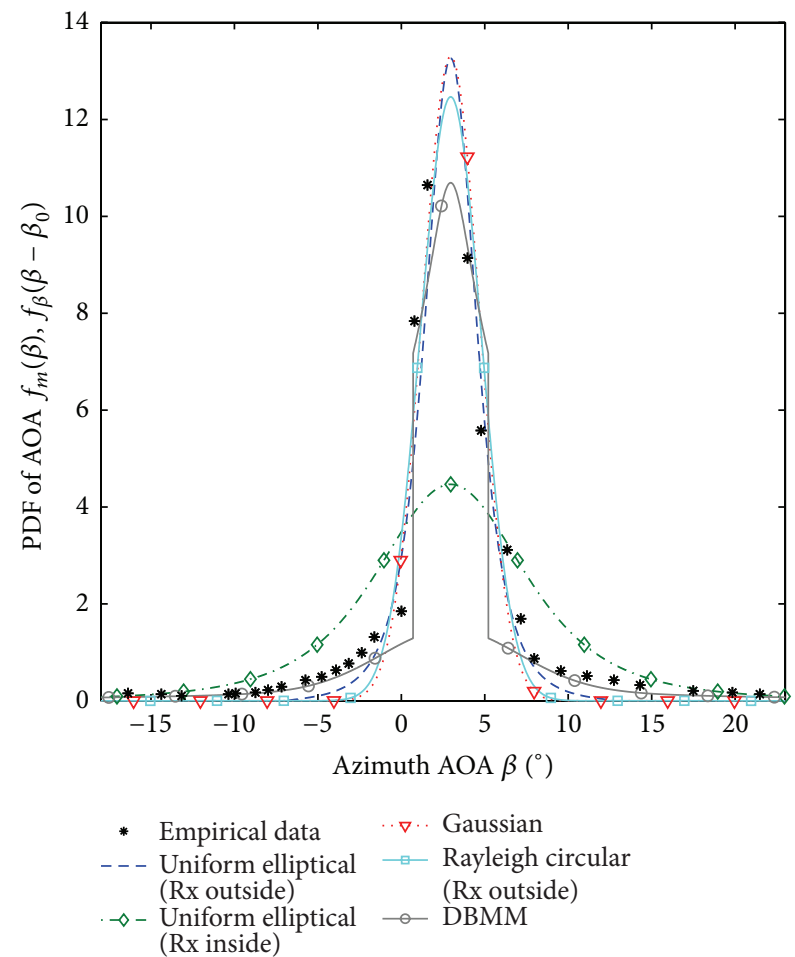

Figure 7: Fitting for various geometric models to the empirical data in Takada et al. [26, Figure 4].

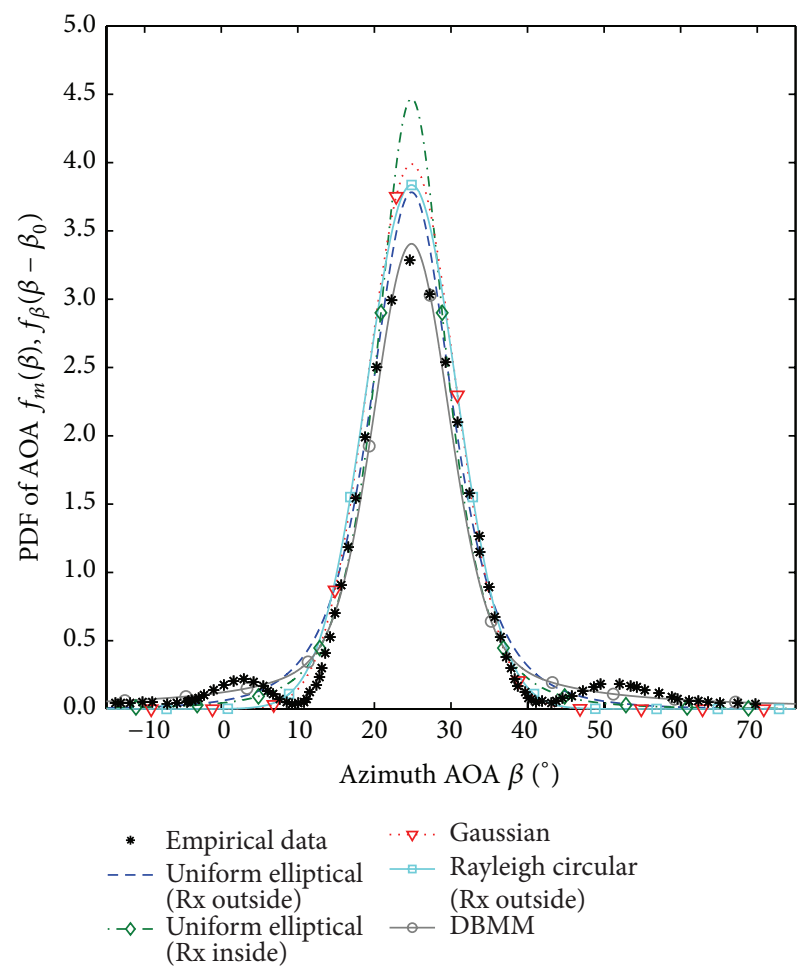

FIGURE 8: Fitting for various geometric models to the empirical data in Fleury et al. [29, Figure 16].

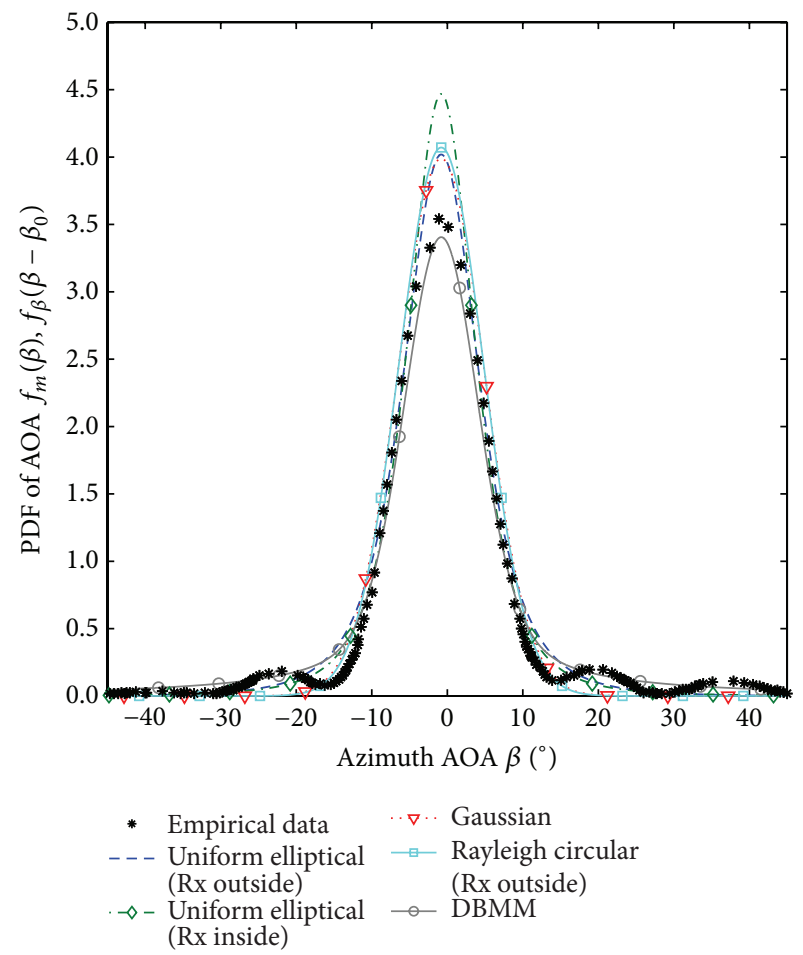

FIGURE 9: Fitting for various geometric models to the empirical data in Mogensen et al. [30, Figure 3]. 


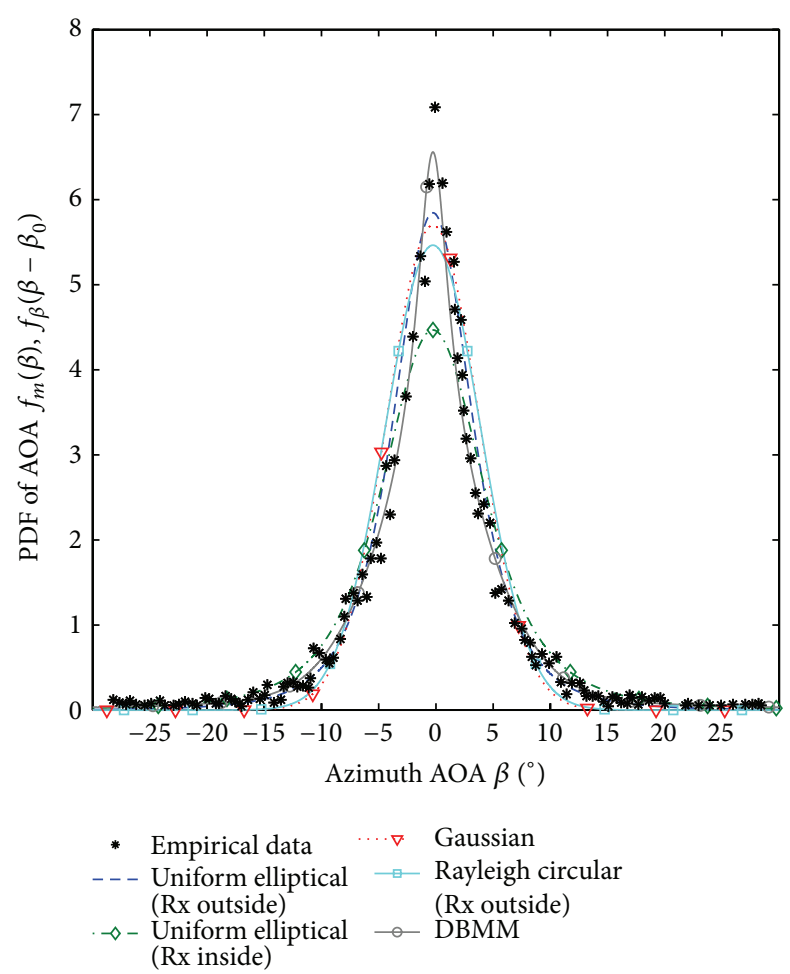

FIGURE 10: Fitting for various geometric models to the empirical data in Pedersen et al. [1, Figure 4], Aarhus.

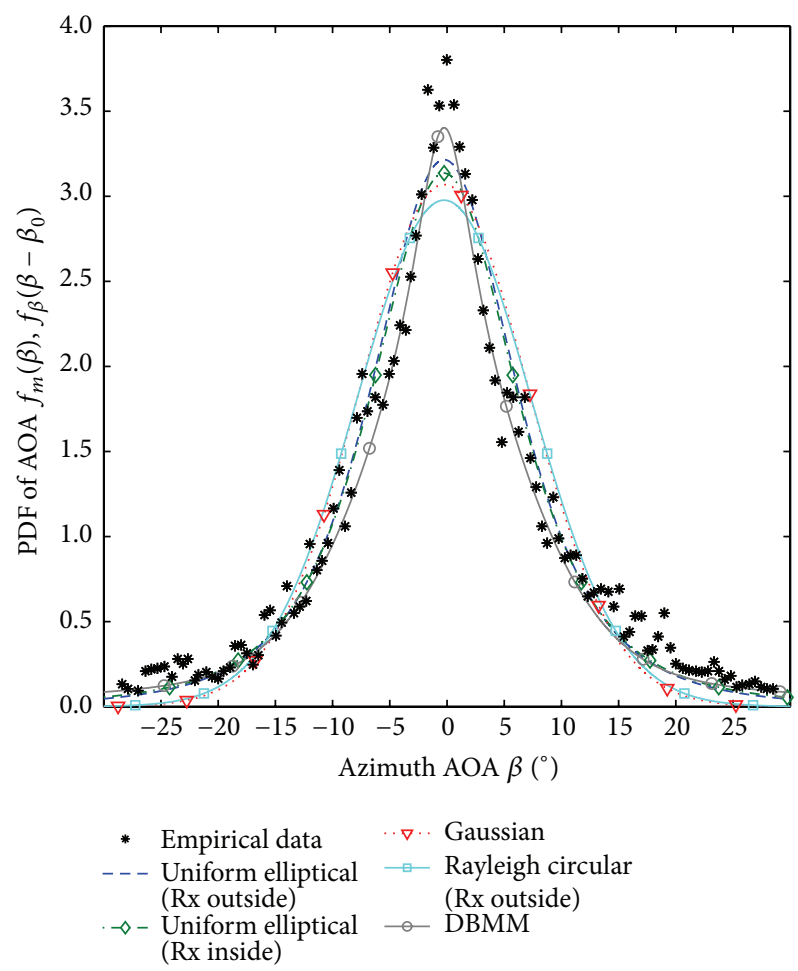

FIGURE 11: Fitting for various geometric models to the empirical data in Pedersen et al. [1, Figure 4], Stockholm.

\section{Conclusion}

In this paper, we presented analytical PDF model of AOA that takes into account the propagation properties of the environment on the basis of the temporal characteristics of channel. In the developed model (DBMM), PDFs of AOA for local and delayed scattering components are modeled by von Mises' PDF and the distribution that results from geometry of the multielliptical propagation model. The parameters of each ellipse are defined on the basis of PDS or PDP. As a result, DBMM is closely related to the propagation conditions of environment and provides a flexible fit to the analyzed radio frequency range. For a given type of environment, DBMM is the spatial estimator of PDF of AOA, because the change of $\mathrm{Tx} / \mathrm{Rx}$ position significantly affects the PDS and PDP. Therefore, the parameters of these characteristics are the estimation parameters of DBMM. Based on the measurement results presented in the literature, the mapping accuracy of the statistical properties of AOA was analyzed for varied propagation scenarios. To compare models, LSE and $D_{m}$ were adopted as a measure of the approximation and estimation accuracy, respectively. The obtained results show that DBMM provides the smallest LSE and $D_{m}$ with respect to other models for all scenarios. This means that DBMM enables the estimation of PDF of AOA for different type of environments based on temporal channel characteristics and description of the propagation scenarios. In practice, the analytical simplicity of the model ensures its easy implementation for simulation studies that take into account the spatial propagation conditions in wireless channels. DBMM is the analytical description of the statistical properties of the reception angle in the Doppler multielliptical channel model (DMCM) [36, 37].

The current trend in the development of channel models goes toward 3D modeling as it is shown in numerous papers, for example, [4,11-13]. The presented model is limited to the azimuth plane, so in the near future, it will be used as a basis for the development of a 3D model.

\section{Conflict of Interests}

The authors declare that there is no conflict of interests regarding the publication of this paper.

\section{References}

[1] K. I. Pedersen, P. E. Mogensen, and B. H. Fleury, "A stochastic model of the temporal and azimuthal dispersion seen at the base station in outdoor propagation environments," IEEE Transactions on Vehicular Technology, vol. 49, no. 2, pp. 437-447, 2000.

[2] T. Aulin, "A modified model for the fading signal at a mobile radio channel," IEEE Transactions on Vehicular Technology, vol. 28, no. 3, pp. 182-203, 1979.

[3] J. D. Parsons and A. M. D. Turkmani, "Characterisation of mobile radio signals: model description," IEE Proceedings I (Communications, Speech and Vision), vol. 138, no. 6, pp. 549556, 1991.

[4] R. Janaswamy, "Angle and time of arrival statistics for the Gaussian scatter density model," IEEE Transactions on Wireless Communications, vol. 1, no. 3, pp. 488-497, 2002. 
[5] L. Jiang and S. Y. Tan, "Geometrically based statistical channel models for outdoor and indoor propagation environments," IEEE Transactions on Vehicular Technology, vol. 56, no. 6, pp. 3587-3593, 2007.

[6] K. N. Le, "On angle-of-arrival and time-of-arrival statistics of geometric scattering channels," IEEE Transactions on Vehicular Technology, vol. 58, no. 8, pp. 4257-4264, 2009.

[7] IST-WINNER II, “WINNER II channel models," IST-WINNER II Tech. Rep. Deliverable 1.1.2 v.1.2., 2007.

[8] K. B. Baltzis, "A generalized elliptical scattering model for the spatial characteristics of mobile channels," Wireless Personal Communications, vol. 67, no. 4, pp. 971-984, 2011.

[9] L. Jiang and S. Y. Tan, "Simple geometrical-based AOA model for mobile communication systems," Electronics Letters, vol. 40, no. 19, pp. 1203-1205, 2004.

[10] A. Y. Olenko, K. T. Wong, and E. H.-O. Ng, "Analytically derived TOA-DOA statistics of uplink/downlink wireless multipaths arisen from scatterers on a hollow-disc around the mobile," IEEE Antennas and Wireless Propagation Letters, vol. 2, no. 1, pp. 345-348, 2003.

[11] A. Y. Olenko, K. T. Wong, S. A. Qasmi, and J. AhmadiShokouh, "Analytically derived uplink/downlink TOA and 2D-DOA distributions with scatterers in a 3-D hemispheroid surrounding the mobile," IEEE Transactions on Antennas and Propagation, vol. 54, no. 9, pp. 2446-2454, 2006.

[12] S. J. Nawaz, N. M. Khan, M. N. Patwary, and M. Moniri, "Effect of directional antenna on the doppler spectrum in 3-Doppler mobile radio propagation environment," IEEE Transactions on Vehicular Technology, vol. 60, no. 7, pp. 2895-2903, 2011.

[13] S. J. Nawaz, M. Riaz, N. M. Khan, and S. Wyne, "Temporal analysis of a 3D ellipsoid channel model for the vehicleto-vehicle communication environments," Wireless Personal Communications, vol. 82, no. 3, pp. 1337-1350, 2015.

[14] A. Ahmed, S. J. Nawaz, and S. M. Gulfam, "A 3-D propagation model for emerging land mobile radio cellular environments," PLOS ONE, vol. 10, no. 8, Article ID e0132555, 2015.

[15] P. Petrus, J. H. Reed, and T. S. Rappaport, "Geometrical-based statistical macrocell channel model for mobile environments," IEEE Transactions on Communications, vol. 50, no. 3, pp. 495$502,2002$.

[16] R. B. Ertel and J. H. Reed, "Angle and time of arrival statistics for circular and elliptical scattering models," IEEE Journal on Selected Areas in Communications, vol. 17, no. 11, pp. 1829-1840, 1999.

[17] N. M. Khan, M. T. Simsim, and P. B. Rapajic, "A generalized model for the spatial characteristics of the cellular mobile channel," IEEE Transactions on Vehicular Technology, vol. 57, no. 1, pp. 22-37, 2008.

[18] A. Abdi, J. A. Barger, and M. Kaveh, "A parametric model for the distribution of the angle of arrival and the associated correlation function and power spectrum at the mobile station," IEEE Transactions on Vehicular Technology, vol. 51, no. 3, pp. 425-434, 2002.

[19] J. D. Parsons and A. S. Bajwa, "Wideband characterisation of fading mobile radio channels," IEE Proceedings-Part F: Communications, radar and signal processing, vol. 129, no. 2, pp. 95-101, 1982.

[20] R. H. Clarke, "A statistical theory of mobile-radio reception," The Bell System Technical Journal, vol. 47, no. 6, pp. 957-1000, 1968.
[21] J. C. Liberti and T. S. Rappaport, Smart Antennas for Wireless Communications: IS-95 and Third Generation CDMA Applications, Prentice Hall PTR, Upper Saddle River, NJ, USA, 1999.

[22] P. C. F. Eggers, "Generation of base station DOA distributions by Jacobi transformation of scattering areas," Electronics Letters, vol. 34, no. 1, pp. 24-26, 1998.

[23] K. T. Wong, Y. I. Wu, and M. Abdulla, "Landmobile radiowave multipaths' DOA-distribution: assessing geometric models by the open literature's empirical datasets," IEEE Transactions on Antennas and Propagation, vol. 58, no. 3, pp. 946-958, 2010.

[24] J. Fühl, J.-P. Rossi, and E. Bonek, "High-resolution 3-d direction-of-arrival determination for urban mobile radio," IEEE Transactions on Antennas and Propagation, vol. 45, no. 4, pp. 672-682, 1997.

[25] A. Kuchar, J.-P. Rossi, and E. Bonek, "Directional macrocell channel characterization from urban measurements," IEEE Transactions on Antennas and Propagation, vol. 48, no. 2, pp. 137-146, 2000.

[26] J.-I. Takada, J. Fu, H. Zhu, and T. Kobayashi, "Spatio-temporal channel characterization in a suburban non line-of-sight microcellular environment," IEEE Journal on Selected Areas in Communications, vol. 20, no. 3, pp. 532-538, 2002.

[27] C. Ziółkowski, "Statistical model of the angular power distribution for wireless multipath environments," IET Microwaves, Antennas \& Propagation, vol. 9, no. 3, pp. 281-289, 2015.

[28] A. Kuchar, M. Taferner, M. Tangemann, and C. Hoek, "Field trial with a GSM/DCS1800 smart antenna base station," in Proceedings of the IEEE VTS Fall 50th Vehicular Technology Conference (VTC 99), vol. 1, pp. 42-46, IEEE, Amsterdam, The Netherlands, 1999.

[29] B. H. Fleury, M. Tschudin, R. Heddergott, D. Dahlhaus, and K. I. Pedersen, "Channel parameter estimation in mobile radio environments using the SAGE algorithm," IEEE Journal on Selected Areas in Communications, vol. 17, no. 3, pp. 434-450, 1999.

[30] P. E. Mogensen, K. I. Pedersen, P. Leth-Espensen et al., "Preliminary measurement results from an adaptive antenna array testbed for GSM/UMTS," in Proceedings of the 47th IEEE Vehicular Technology Conference (IEEE VTC '97), vol. 3, pp. 1592-1596, IEEE, Phoenix, Ariz, USA, May 1997.

[31] A. Rohatgi, "WebPlotDigitizer 3.8," May 2015, http://arohatgi .info/WebPlotDigitizer/.

[32] V. K. Rohatgi and A. K. M. E. Saleh, An Introduction to Probability and Statistics, Wiley-Interscience, New York, NY, USA, 2nd edition, 2000.

[33] K. I. Pedersen, P. E. Mogensen, and B. H. Fleury, "Spatial channel characteristics in outdoor environments and their impact on BS antenna system performance," in Proceedings of the 48th IEEE Vehicular Technology Conference (IEEE VTC '98), vol. 2, pp. 719-723, Ottawa, Canada, 1998.

[34] P. A. Matthews, D. Molkdar, and B. Mohebbi, "Direction of arrival and frequency response measurements at UHF," in Proceedings of the 5th International Conference on Mobile Radio and Personal Communications, pp. 43-47, Coventry, UK, December 1989.

[35] M. Failli, "COST 207. Digital land mobile radio communications," Final Report COST 207 (1984.03.14.-1988.09.13), Commission of the European Communities, Directorate-General Telecommunications, Information Industries and Innovation, Luxembourg City, Luxembourg, 1989.

[36] C. Ziółkowski and J. M. Kelner, "Geometry-based statistical model for the temporal, spectral, and spatial characteristics of 
the land mobile channel," Wireless Personal Communications, vol. 83, no. 1, pp. 631-652, 2015.

[37] C. Ziółkowski and J. M. Kelner, “DMCM ver. 2.0,” Doppler Multi-Elliptical Channel Model, 2014, http://www.dmcm.org .pl/.

[38] R. J. Piechocki, G. V. Tsoulos, and J. P. McGeehan, "Simple general formula for PDF of angle of arrival in large cell operational environments," Electronics Letters, vol. 34, no. 18, pp. 1784-1785, 1998.

[39] J. C. Liberti and T. S. Rappaport, "A geometrically based model for line-of-sight multipath radio channels," in Proceedings of the 46th IEEE Vehicular Technology Conference. Mobile Technology for the Human Race, vol. 2, pp. 844-848, IEEE, Atlanta, Ga, USA, April-May 1996.

[40] A. Andrade and D. Covarrubias, "Radio channel spatial propagation model for mobile 3G in smart antenna systems," IEICE Transactions on Communications, vol. E86-B, no. 1, pp. 213-220, 2003.

[41] D. D. N. Bevan, A. G. Flaksman, V. T. Ermolayev, and I. M. Averin, "Gaussian channel model for mobile multipath environment," Eurasip Journal on Applied Signal Processing, vol. 2004, no. 9, pp. 1321-1329, 2004.

[42] J. Laurila, A. F. Molisch, and E. Bonek, "Influence of the scatterer distribution on power delay profiles and azimuthal power spectra of mobile radio channels," in Proceedings of the 5th IEEE International Symposium on Spread Spectrum Techniques and Applications, vol. 1, pp. 267-271, IEEE, Sun City, South Africa, September 1998. 

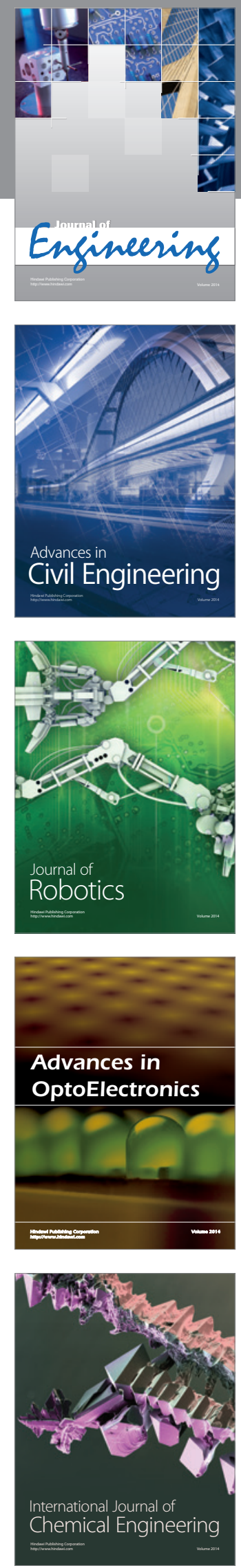

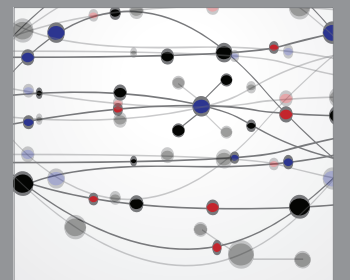

The Scientific World Journal
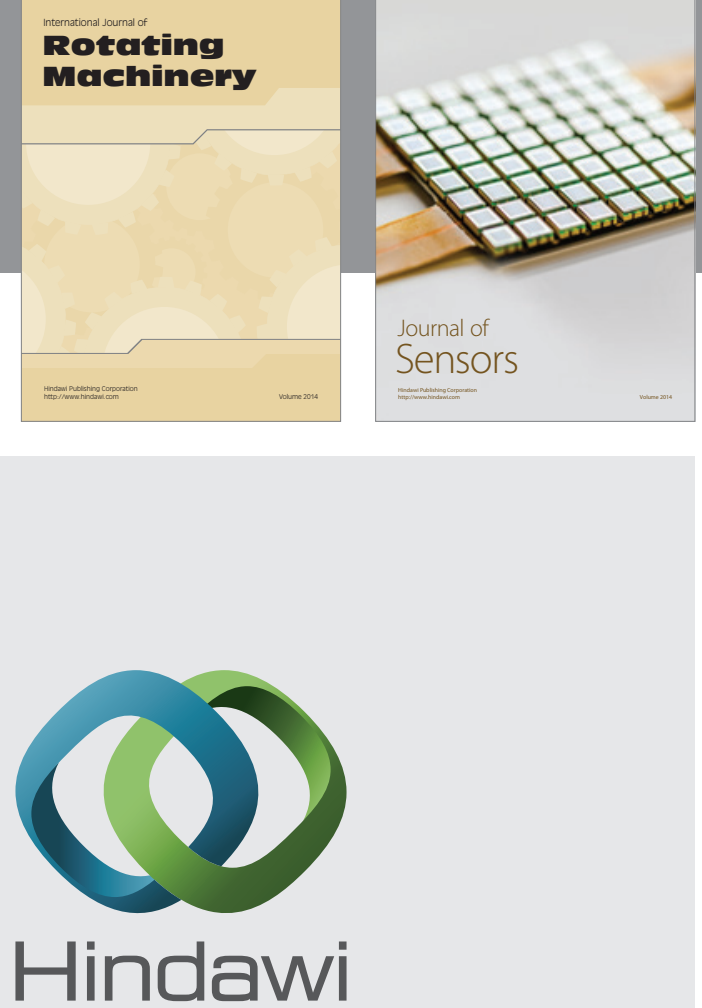

Submit your manuscripts at http://www.hindawi.com
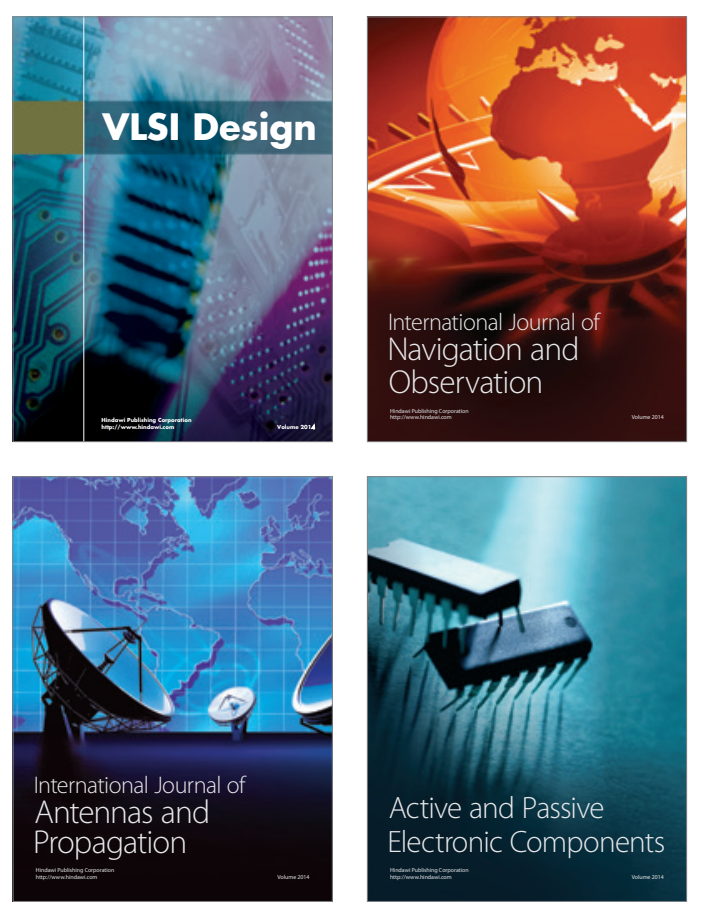
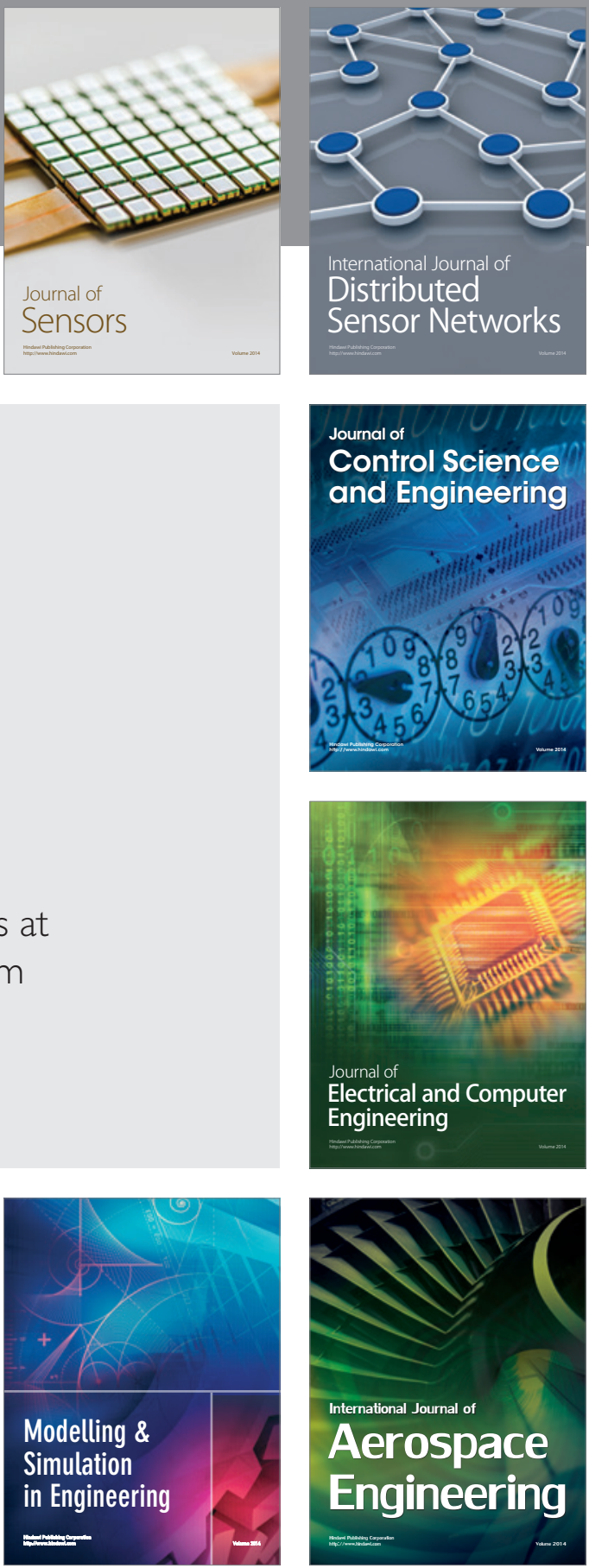

Journal of

Control Science

and Engineering
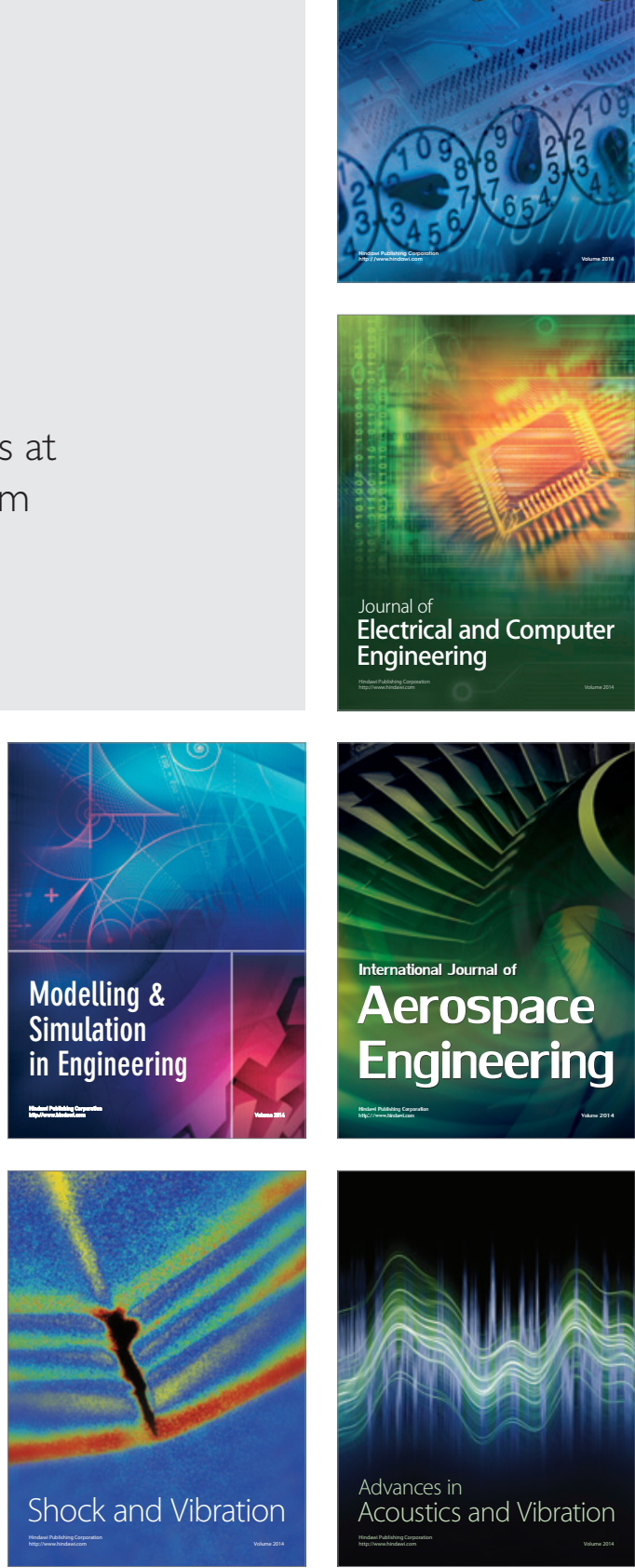